\title{
Looking Within Rather Than Between Countries to Understand the Risk Factors for Vision Impairment
}

\author{
Rupert R. A. Bourne, FRCOphth, MD; Jost B. Jonas, MD; Serge Resnikoff, MD, PhD
}

In this issue of JAMA Ophthalmology, an article by Ehrlich et al 1 reports on the vision impairment findings of the World Health Organization's Study on Global Aging and Adult Health (SAGE) study. The SAGE study is a longitudinal research project collecting nationally representative data on adults 50 years and older and a smaller comparison sample of adults aged 18 to 49 years in China, Ghana, India, Mexico, the Russian Federation, and South Africa. The first wave of data collection occurred between 2007 and 2010. ${ }^{2}$ Because the study was supported by the US National Institute on Aging, Division of Behavioral and Social Research, national governments, and the World Health Organization, the microdata and weighted metadata are in the public domain. Ehrlich et al ${ }^{1}$ used these data to look at a series of nonophthalmological factors associated with presenting impairments in distance and near vision in each country; these included health, well-being, demographics, and the socioeconomic circumstances of participants and their households. There have been relatively few multicountry studies that combine measured vision loss and risk factors in older adults, and this study sheds an important light on these associations within low- and-middleincome countries (which make up $84 \%$ of the global population). There are several reasons why this article is worthy of comment.

The countries involved represent an important opportunity to address the sparseness of distance vision loss prevalence data from large sections of the world. For example, a large meta-analysis of population-based eye surveys called the Global Vision Database (GVD) reported that there had been only 5 high-quality studies on vision published in China between 1980 and 1999, with 33 such studies published between 2000 and 2014. The numbers of studies in the GVD for these periods number 0 and 2, respectively, in Ghana, 5 and 13 in India, 0 and 1 in the Russian Federation, and 0 and 2 in Mexico. Nationally representative eye surveys are uncommon; only 44 of the 288 GVD study sources (15.8\%), for example, were national in scope.

Although the article ${ }^{1}$ does not report actual prevalence rates of distance vision impairment (defined as presenting visual acuity of $<6 / 18$, an approximate Snellen equivalent of less than $20 / 60$, in the better eye) but rather reports the proportion of the sample affected, the completeness of the visual acuity data coverage (which exceeded $70 \%$ in most countries) and the relatively large sample sizes lend credence to the very substantial burden of distance vision impairment in these countries. This burden ranges from $10 \%$ in China and South Africa to $25 \%$ in the Russian Federation. Additionally, the near-vision loss burden is very large, ranging from $28 \%$ in Ghana to $43 \%$ in India. More recently, there have been some local studies in the Russian Federation, ${ }^{3}$ yet this work highlights the importance of improving understanding of vision loss burden in Central and Eastern Europe, as well as Latin America, where data sources are too scarce.

It comes as no surprise that the age structure of populations makes a considerable difference to overall burden of distance vision loss and in all countries studied; in the article by Ehrlich et al, 1 higher age and self-reported disability were significantly associated with distance vision impairment. Yet this study also showed that in most countries, poverty (measured in SAGE as a household wealth index), reduced physical functioning, comorbidities, cognitive impairment (as measured by memory function tested by verbal recall), and social isolation were also associated. Many of these factors of course are becoming increasingly common in aging populations.

Country, regional, and global estimates of the prevalence of vision impairment are clearly of great importance, particularly in advocacy and for monitoring changes over time. The Vision Loss Expert Group's Global Vision Database and the associated Vision Atlas project,,-4 which is made possible by a 
collaboration with the International Agency for Prevention of Blindness, go a long way toward making these data more accessible. The estimated burdens of vision impairment within each country by age and sex provided by these projects provide the rationale for scaling up eye care interventions.., 6 Yet as the period of the World Health Organization's Global Action Plan (2014-2019) comes to an end and there is recognition that its target of a $25 \%$ reduction in avoidable blindness has not been met yet (owing largely to the aging of populations), perhaps the global eye care community should now set its sights on the inequity within countries and the factors that could be addressed to reduce these.

Although there are limitations of the SAGE survey (such as not analyzing the ophthalmological causes of vision impairment; a relatively low participation rate of $70.5 \%$ and $72.9 \%$ in the Russian Federation for distance and near vision assessments, respectively; the involvement of proxy respondents; and a process of interviewing only a very small proportion of the population, which may miss high-risk groups $\underline{\underline{I}}$ and reduce population representativeness), this study $\underline{\underline{1}}$ has illustrated the heterogeneity in the factors that are associated with vision loss by country. Another important factor is the availability of health insurance among respondents of these surveys and whether this covered eye care. Yet these details was not reported by Ehrlich et al, $\underline{1}$ although these data were collected by the SAGE survey. The proportion of the sample in receipt of an eye examination within the 2 years preceding the survey varied considerably between countries, ranging from $15 \%$ in Ghana to $53 \%$ in the Russian Federation. This is also of interest.

Each country faces a unique set of environmental, social, and economic circumstances that are risk factors for late presentation of eye disease, and these are by no means limited to those of low- and middle-income countries. A major interest of this study 1 is to provide information on in-country granularity of the associations that local socioeconomics and demographics have with vision impairment and receipt of eye care. The issue here is that in very large and diverse countries, such as China, the Russia Federation, and India, country-wide sampling may mask statistically significant differences between provinces, regions, and/or states. In other words, the intercountry heterogeneity of associations observed among countries may also exist within countries (eg, between Eastern and Western China), and this should be taken into account when planning future similar surveys.

Detailed knowledge of these factors within countries would open up the opportunity to trial interventions more effectively not just by traditional eye care professionals but also in association with allied groups, such as educationalists, social care groups, specialists in diseases of elderly individuals (such as dementia and hearing loss), and economists. These efforts require in-country collaboration between governmental and nongovernmental organizations and patient and public participation. There are already many excellent examples of such programs in developing countries. ${ }^{-}$Indeed, the ophthalmology community working among these other professionals offers the opportunity to raise awareness of the unacceptably high burden of vision loss and bring collaborative solutions tailor-made for individual countries and delivered in-country.

Corresponding Author: Rupert R. A. Bourne, FRCOphth, MD, Vision \& Eye Research Unit, Anglia Ruskin University, East Road, Cambridge CB1 1PT, United Kingdom (rb@rupertbourne.co.uk).

Conflict of Interest Disclosures: Dr Jonas reports acting as a consultant for Mundipharma Co, holding a patent with Biocompatibles UK Ltd (No. 20120263794), and holding a patent application with University of Heidelberg (Europäische Patentanmeldung 15000 771.4). Dr Resnikoff reports serving as a consultant for Brien Holden Vision Institute. Dr Bourne reports having received advisory board, speaker fees, and consultancy fees from GlaxoSmithKline, Thea, Santen, and Allergan. No other disclosures were reported. 


\section{References:}

1.

Ehrlich JR, Stagg BC, Andrews C, Kumagai A, Musch DC. Vision impairment and receipt of eye care among older adults in low- and middle-income countries [published online November 21,

2018]. JAMA Ophthalmol. doi:10.1001/jamaophthalmol.2018.5449

2.

World Health Organization. World Health Organization Study on Global Ageing and Adult Health (SAGE). http://www.who.int/healthinfo/sage/en/. Published 2018. Accessed September 13, 2018.

3.

Bikbov M, Fayzrakhmanov RR, Kazakbaeva G, Jonas JB. Ural Eye and Medical Study: description of study design and methodology. Ophthalmic Epidemiol. 2018;25(3):187-198.

doi: $10.1080 / 09286586.2017 .1384504$

4.

International Agency for the Prevention of Blindness. Vision atlas. http://atlas.iapb.org Published 2018. Accessed September 13, 2018.

5.

Bourne RRA, Flaxman SR, Braithwaite T, et al; Vision Loss Expert Group. Magnitude, temporal trends, and projections of the global prevalence of blindness and distance and near vision impairment: a systematic review and meta-analysis. Lancet Glob Health. 2017;5(9):e888-e897. doi:10.1016/S2214-109X(17)30293-0

6.

Flaxman SR, Bourne RRA, Resnikoff S, et al; Vision Loss Expert Group of the Global Burden of Disease Study. Global causes of blindness and distance vision impairment 1990-2020: a systematic review and meta-analysis. Lancet Glob Health. 2017;5(12):e1221-e1234. doi:10.1016/S2214$\underline{109 \times(17) 30393-5}$

7.

Foreman J, Keel S, van Wijngaarden P, et al. Prevalence and causes of visual loss among the indigenous peoples of the world: a systematic review. JAMA Ophthalmol. 2018;136(5):567-580. doi:10.1001/jamaophthalmol.2018.0597

8.

Congdon N, Yan X, Lansingh V, et al. Assessment of cataract surgical outcomes in settings where follow-up is poor: PRECOG, a multicentre observational study. Lancet Glob Health. 2013;1(1):e37e45. doi:10.1016/S2214-109X(13)70003-2 